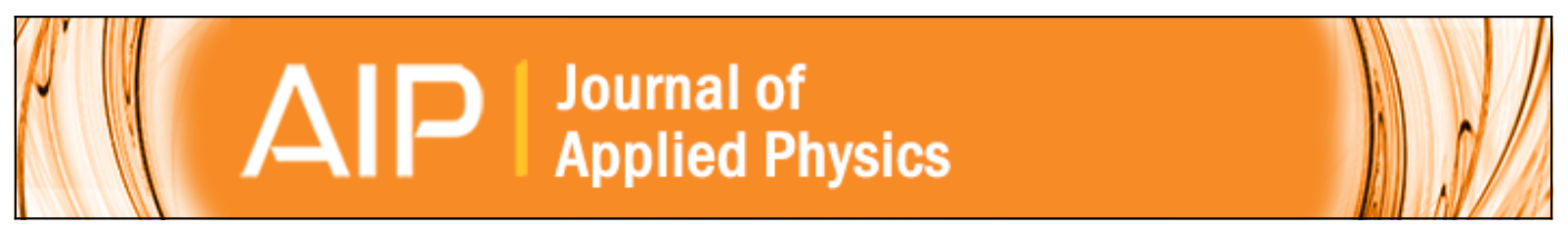

\title{
Coherent thermoelectric transport in single, double, and U-bend structures
}

A. J. Pye, D. A. Faux, and M. J. Kearney

Citation: Journal of Applied Physics 117, 064312 (2015); doi: 10.1063/1.4907911

View online: http://dx.doi.org/10.1063/1.4907911

View Table of Contents: http://scitation.aip.org/content/aip/journal/jap/117/6?ver=pdfcov

Published by the AIP Publishing

\section{Articles you may be interested in}

Ballistic thermoelectric properties in boron nitride nanoribbons

J. Appl. Phys. 114, 144311 (2013); 10.1063/1.4824750

Ballistic thermoelectric properties in graphene-nanoribbon-based heterojunctions

Appl. Phys. Lett. 101, 103115 (2012); 10.1063/1.4751287

Geometrical effects on the thermoelectric properties of ballistic graphene antidot lattices

J. Appl. Phys. 110, 054506 (2011); 10.1063/1.3629990

Ballistic thermoelectricity in double-bend nanowires

Appl. Phys. Lett. 98, 173107 (2011); 10.1063/1.3583670

Acoustic phonon transport and ballistic thermal conductance through a three-dimensional double-bend quantum structure

J. Appl. Phys. 104, 054312 (2008); 10.1063/1.2975979

Frustrated by

old technology?

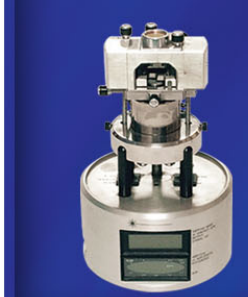

Is your AFM dead

and can't be repaired?

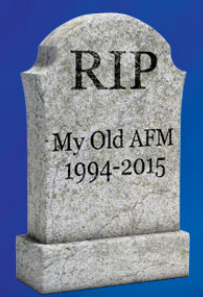

Sick of bad customer support?

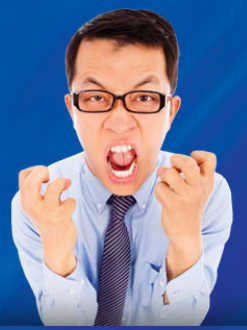

It is time to upgrade your AFM

Minimum $\$ 20,000$ trade-in discount for purchases before August 31st

Asylum Research is today's technology leader in AFM

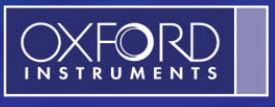




\title{
Coherent thermoelectric transport in single, double, and U-bend structures
}

\author{
A. J. Pye, D. A. Faux, and M. J. Kearney \\ Faculty of Engineering and Physical Sciences, University of Surrey, Guildford, Surrey GU2 7XH, \\ United Kingdom
}

(Received 25 September 2014; accepted 30 January 2015; published online 11 February 2015)

\begin{abstract}
Coherent, i.e., ballistic, thermoelectric transport in electron waveguide structures containing right-angle bends in single, double, and U-bend configurations is investigated. A theory based on Green's functions is used to derive the transmission function (and from that the transport coefficients) and allows for the inclusion of realistic models of spatially distributed imperfections. The results for the single and double-bend structures are presented in more detail than elsewhere in the literature. In the U-bend structure, sharp resonances in the stop-band region of the transmission function lead to large-magnitude peaks in the thermopower and consequently a large thermoelectric figure of merit (of order ten in some instances). These properties are still readily apparent even in the presence of moderate edge roughness or Anderson disorder. (C) 2015 AIP Publishing LLC.
\end{abstract}

[http://dx.doi.org/10.1063/1.4907911]

\section{INTRODUCTION}

The field of quantum transport has seen a great deal of interest since the observation of quantized electrical conductance was first reported. ${ }^{1,2}$ In the early work in this area, quantum point contacts were used to achieve the small width necessary for conductance quantization to be apparent. Subsequently, it has become relatively straightforward to fabricate "electron waveguides," which are narrow enough to show quantum size effects.

Electron waveguides have been the subject of much investigation, primarily because many different structures can be produced by modifying the waveguide. Examples include the theoretical investigation of straight waveguides with sections of mismatched widths ${ }^{3}$ and single and double right-angle bend waveguides. ${ }^{4}$ The investigations of the double bend showed that the transmission function contains resonant structure, which was later confirmed experimentally. ${ }^{5}$ Following initial studies of the double bend, the effects of Anderson disorder, ${ }^{6}$ edge roughness, and isolated impurities were investigated. ${ }^{7}$ The transmission function for a series of two and three double bends was calculated and the effect of rounded corners in the bend regions and bends of angles other than $90^{\circ}$ were examined. ${ }^{6,7}$

Quantum size effects are predicted to enhance thermoelectric performance ${ }^{8}$ and for this reason the thermoelectric properties of these waveguide structures are of interest. The first reported observation of thermoelectric effects in a double bend ${ }^{9}$ suggested that the structure could show stronger thermoelectric behavior than a quantum point contact. The thermopower of a double bend has also been calculated numerically ${ }^{10}$ with the results showing that the resonant structure of the transmission function gives rise to large magnitudes in the thermopower. A complete understanding of the thermoelectric properties of such structures requires an understanding of the role played by phonons; to this end, calculations of the phonon contribution to the thermal conductivity have also been undertaken for double bends. ${ }^{11,12}$ The thermal conductivity is one of the factors which influences the overall thermoelectric figure of merit and the reduction of the phonon contribution due to geometric constraints, as well as due to the low operating temperatures typically employed, is seen as advantageous. This has recently been explored in the context of generic one-dimensional nanostructures, ${ }^{13}$ $\mathrm{Si}_{1-x} \mathrm{Ge}_{x}$ nanowires, ${ }^{14}$ and graphene nano-ribbons. ${ }^{15}$ Phonons also play a role in modifying the thermopower through the effects of scattering and through the mechanism of phonon drag (see, for example, Ref. 16). Again, evidence suggests that at sufficiently low temperatures the purely electronic component can dominate in waveguide structures and therefore studies which neglect phonons are relevant as regards defining limiting behavior (a recent review of all these considerations may be found in Ref. 17). In this work, we examine single, double, and U-bend structures, and in the case of the latter we pay particular attention to the effects of disorder or imperfections of various kinds. As far as we are aware, the Ubend investigated here has not been studied elsewhere. One similar structure appears in the literature, but this is attached to leads much wider than the waveguide itself, ${ }^{18}$ introducing additional ringing in the transmission function, ${ }^{19,20}$ which is not present in our structures.

The paper is organised as follows. In Sec. II, the theory used to model the single, double, and U-bend electron waveguides is outlined. In Sec. III, the transport characteristics of these structures, including thermoelectric effects and the impact of imperfections, are investigated. Finally, in Sec. IV, we summarize the work and present our concluding remarks.

\section{THEORY}

In this section, the theory used to model the waveguide structures is presented. Our approach is relatively standard and is based on the well-established Landauer-Büttiker picture of quantum transport, ${ }^{21-24}$ of which an excellent overview may be found in Ref. 25. However, whilst our overall approach is not novel in itself, we take the opportunity here to discuss some specific theoretical aspects where these have 
not been presented previously in the literature in any particular detail.

For the purposes of modelling, the "system" of interest consists of a rectangular region which is connected to two leads; see Fig. 1. The device structure itself is defined by specifying exclusion regions within the rectangular region by the use of a large potential. Each lead is assumed to be independent, semi-infinite, multi-moded and terminates in a reservoir far away from the device. Each reservoir is assumed to be large enough that the addition or removal of a carrier will not affect its internal state. An individual reservoir therefore provides a source and sink of carriers in its associated lead, with the carrier states maintained at a local equilibrium temperature and chemical potential along the length of the lead. In this model, all equilibration occurs in the reservoirs and the carriers maintain their phase coherence within the device itself. These fundamental assumptions are those which underpin the Landauer-Büttiker modelling approach ${ }^{21-24}$ and allow the device to be studied as part of an open quantum system-a necessity for transport calculations.

In what follows, these assumptions are applied to the three geometries shown in Fig. 1. Note that a length $d$ of each of the leads is included as part of the device itself; this is necessary to ensure proper treatment of the bends (as discussed later). The $z$-direction is assumed to decouple from the $x$ - and $y$-directions leaving a quasi-two-dimensional real space. This is discretized using a grid with $N_{x}$ and $N_{y}$ points in the $x$ - and $y$-directions, respectively, with grid spacings of $a_{x}$ and $a_{y}$. This provides $N=N_{x} N_{y}$ points in the device space and we refer to each point on the two-dimensional grid by a single index $n$ or $m$. A standard Green's function formalism $^{25}$ is used to evaluate the transport properties of the device, the starting point of which is the timeindependent Schrödinger equation for electrons of effective mass $m^{*}$ given by

$$
\left[-\frac{\hbar^{2}}{2 m^{*}} \nabla^{2}+U(x, y)\right] \psi(x, y)=E \psi(x, y),
$$

where $U(x, y)$ is the potential. This includes the aforementioned exclusion regions (which define the waveguide geometry) and also allows for imperfections such as edge roughness and Anderson disorder to be added in, as discussed later.

To solve Eq. (1) numerically, a centered finitedifference method is used to discretize the equation yielding an $N \times N$ Hamiltonian matrix, which captures the physics of the device. The elements of this matrix are given by

$$
[\hat{H}]_{n m}= \begin{cases}2\left(t_{x}+t_{y}\right)+U_{n}, & n=m \text { (on-site energy), } \\ -t_{x}, & n \text { and } m x \text {-adjacent, } \\ -t_{y}, & n \text { and } m y \text {-adjacent } \\ 0, & \text { otherwise }\end{cases}
$$

where $t_{x, y}=\hbar^{2} / 2 m^{*} a_{x, y}^{2}$, and $U_{n}$ denotes the value of the potential taken at point $n$ on the grid. Ultimately, the derived matrices of interest which enable the calculation of transport coefficients correspond to the retarded and advanced Green's functions for the whole leads-plus-device system, written, respectively, as $G^{R}$ and $G^{A}$. At an energy $E$, these are given by

$$
\begin{gathered}
G^{R}=\frac{1}{a_{x} a_{y}}[(E+i \eta) I-\hat{H}-\Sigma]^{-1} \\
G^{A}=\left[G^{R}\right]^{\dagger}
\end{gathered}
$$

with $\eta$ an infinitesimal positive quantity and the dagger symbolizing the conjugate transpose. Here, $\Sigma$ is a self-energy matrix which captures the effect of the leads coupling to the device and ultimately accounts for the equilibration, which occurs in the reservoirs. A self-energy matrix $\Sigma_{p}$ is calculated for each lead $p=1,2$ and these are then summed to obtain the quantity in Eq. (3) as $\Sigma=\Sigma_{1}+\Sigma_{2}$. This approach allows for the inclusion of semi-infinite leads using only finite-sized matrices on a discretized real-space grid. The appropriate expression for the self-energy matrix for the $p$ th lead is well-known ${ }^{25}$

$$
\Sigma_{p}=a_{x} a_{y}\left[\tau_{d p} g_{p} \tau_{p d}\right]
$$

where $g_{p}$ is the retarded Green's function matrix for the semi-infinite lead in isolation and the $\tau$ are coupling matrices
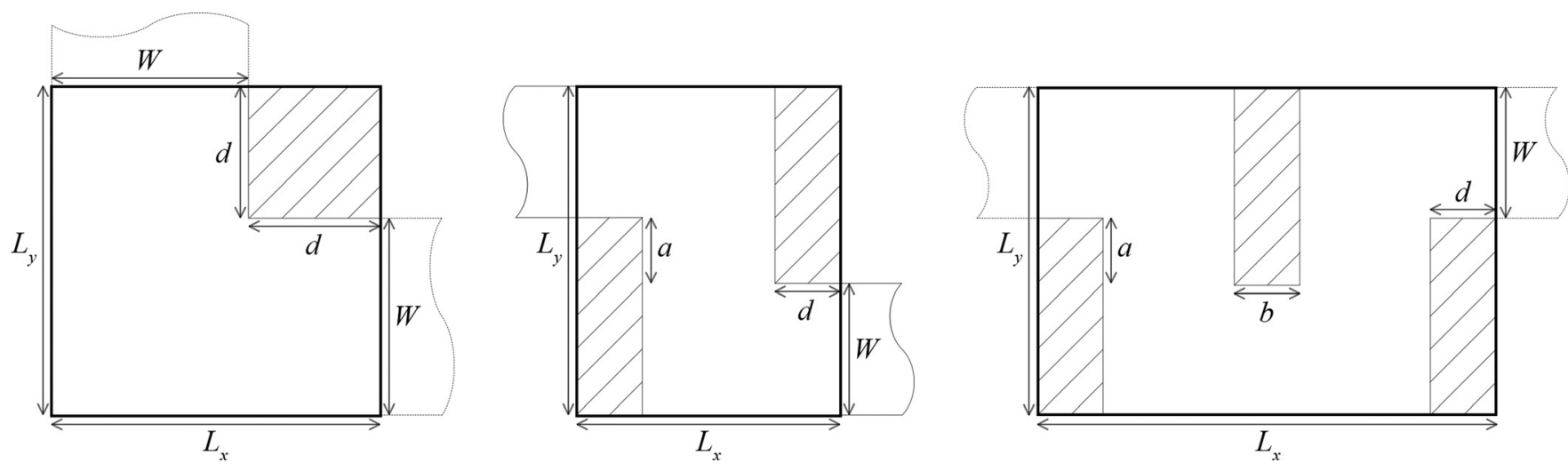

FIG. 1. Depicted from left to right are single, double, and U-bend electron waveguides. The lengths $L_{x}$ and $L_{y}$ are the length and width of the device region, respectively, indicated by the bold line. Hatched regions represent exclusion areas where an arbitrarily large repulsive potential is applied. These regions define the structure itself via the interbend lengths $a$ and $b$, and the length $d$ of each lead in the device region. The width of the waveguide is $W$, and the curved lines indicate the positions of the semi-infinite leads. 
representing the binding between the lead and device. This coupling is assumed to be the same as that between adjacent grid points in the device region enabling the elements of the matrices to be written as

$$
\begin{aligned}
{\left[\tau_{d p}\right]_{n m} } & = \begin{cases}-\delta_{n m} t_{x_{p}}, & \text { point } n \text { adjacent to lead } p \\
0, & \text { otherwise }\end{cases} \\
& =\left[\tau_{p d}\right]_{m n}^{*}
\end{aligned}
$$

where $x_{p}$ is defined in relation to the longitudinal direction of lead $p$. Thus, $x_{p}=x$ for leads connected to the left and right edges of the device region, and $x_{p}=y$ for the lead attached to the top edge of the single bend as illustrated in Fig. 1. The two leads are the same width for convenience.

The nature of the coupling matrices means that it is only necessary to evaluate the lead Green's functions at the end of the lead adjacent to the device. The elements of this surface Green's function are found from the continuous spectral representation,

$$
g_{p}\left(x_{p}, y_{p} ; x_{p}^{\prime}, y_{p}^{\prime} ; E\right)=\sum_{\alpha} \sum_{k_{\alpha}} \frac{\psi_{\alpha, k_{\alpha}}\left(x_{p}, y_{p}\right) \psi_{\alpha, k_{\alpha}}^{*}\left(x_{p}^{\prime}, y_{p}^{\prime}\right)}{E+i \eta-\epsilon_{\alpha, k_{\alpha}}},
$$

where $\psi_{\alpha, k_{\alpha}}$ and $\epsilon_{\alpha, k_{\alpha}}$ are the wavefunction and energy, respectively, for the state with transverse mode index $\alpha$ and longitudinal wavenumber $k_{\alpha}$ in lead $p$. We also set $x_{p}^{\prime}=x_{p}$ since only the surface values of this function are of interest. Each lead is assumed to be straight, semi-infinite in length and have a square-well cross-section given by

$$
U_{p}\left(y_{p}\right)=\left\{\begin{array}{cc}
0, & -\frac{W}{2}<y_{p}<\frac{W}{2}, \\
\infty, & \text { otherwise }
\end{array}\right.
$$

with $y_{p}$ measured relative to the transverse center of lead $p$. The eigenstates of such a lead are

$$
\begin{aligned}
\psi_{\alpha, k_{\alpha}}\left(x_{p}, y_{p}\right) & =\sqrt{2 / L} u_{\alpha}\left(y_{p}\right) e^{i k_{\alpha} x_{p}}, \\
\epsilon_{\alpha, k_{\alpha}} & =\epsilon_{\alpha}+\frac{\hbar^{2} k_{\alpha}^{2}}{2 m^{*}},
\end{aligned}
$$

with

$$
\begin{aligned}
u_{\alpha}\left(y_{p}\right) & =\sqrt{2 / W} \sin \left(\alpha \pi\left(y_{p}+W / 2\right) / W\right), \\
\epsilon_{\alpha} & =\frac{\hbar^{2} \pi^{2} \alpha^{2}}{2 m^{*} W^{2}},
\end{aligned}
$$

and $\alpha=1,2 \ldots$ Substituting Eqs. (9) and (10) into Eq. (7) and discretizing, the lead Green's function matrix elements are obtained as

$$
\left[g_{p}\right]_{n m}=-\frac{2 m^{*} a_{x_{p}}}{\hbar^{2}} \sum_{\alpha}\left[u_{\alpha}\right]_{n}\left[u_{\alpha}^{*}\right]_{m} e^{i k_{\alpha} a_{x_{p}}},
$$

with $\left[u_{\alpha}\right]_{n}$ the value of the $\alpha$ th transverse wavefunction at grid point $n$. The summation runs over the propagating modes $\alpha$ in lead $p$, for which $\epsilon_{\alpha}<E$. These modes are assumed to be dominant, so that evanescent modes can be neglected.
It is intuitively clear that by including a length of the leads as part of the device structure itself, i.e., by spatially removing the interfaces with the semi-infinite leads (where phase coherence is lost), one should improve the accuracy of the modelling. The only limitation is one of the computational processing resources, so in practice a suitable compromise is necessary. In what follows, we have included a length $d$ of each lead in the device region whose value has been determined by testing to see that the results are effectively insensitive to any further increase. In this way, the fact that the states in the waveguide extend slightly beyond the bends themselves is effectively captured and better "matching" occurs with the states supplied by the leads (which are those of a semi-infinite straight wire).

Once the lead self-energies and system Green's functions are known, the transmission probabilities $t_{q p}$ can be calculated. These give the probability of a carrier incident on the device from lead $p$ exiting via lead $q$. The standard approach is to use the Fisher-Lee equation in a continuous multi-moded representation, which gives the scattering or $S$-matrix element in terms of the Green's function at the lead-device interface ${ }^{25,26}$

$$
\begin{aligned}
s_{\beta \alpha}(E)= & -\delta_{\beta \alpha}+i \hbar \sqrt{v_{\beta} v_{\alpha}} \iint u_{\beta}\left(y_{q}\right) G^{R} \\
& \times\left(x_{p}=0, y_{p} ; x_{q}=0, y_{q} ; E\right) u_{\alpha}\left(y_{p}\right) \mathrm{d} y_{q} \mathrm{~d} y_{p} .
\end{aligned}
$$

The $S$-matrix element $s_{\beta \alpha}$ is the fraction of the wave amplitude from mode $\alpha$ in lead $p$ that scatters into mode $\beta$ in lead $q$ and $v_{\beta}$ is the electron velocity in mode $\beta$. The lead-specific local coordinates $x_{p}$ and $y_{p}$ correspond, respectively, to the longitudinal and transverse directions in lead $p$ with the interface between the lead and device positioned at $x_{p}=0$.

The transmission probability (or transmission function) from mode $\alpha$ in lead $p$ to mode $\beta$ in lead $q$ is given by the squared modulus of the relevant $S$-matrix element. By summing over all propagating modes, the total transmission function from lead $p$ to lead $q$ is obtained as

$$
t_{q p}(E)=\sum_{\beta} \sum_{\alpha}\left|s_{\beta \alpha}(E)\right|^{2} \text {. }
$$

The present systems contain only two leads so that $p, q=1,2$. Using Eq. (12) in this expression, simplifying and discretizing, yields

$$
\begin{aligned}
t_{q p}(E)= & \delta_{q p}\left\{M_{p}(E)+2 a_{x} a_{y} \operatorname{Tr}\left[\Gamma_{p}(E) \operatorname{Im}\left\{G^{R}(E)\right\}\right]\right\} \\
& +\left(a_{x} a_{y}\right)^{2} \operatorname{Tr}\left[G^{R}(E) \Gamma_{q}(E) G^{A}(E) \Gamma_{p}(E)\right]
\end{aligned}
$$

where $M_{p}(E)=\sum_{\alpha} 1$ is the number of propagating modes in lead $p$ at energy $E$, Tr and Im are, respectively, the trace and imaginary parts, and

$$
\Gamma_{p}(E)=i\left(\Sigma_{p}(E)-\left[\Sigma_{p}(E)\right]^{\dagger}\right)
$$

is the broadening matrix for lead $p$. This is a measure of the imaginary component of the effective Hamiltonian $\left[\hat{H}-\Sigma_{p}\right]$, which broadens the energy levels of the system and introduces a finite state lifetime corresponding to phase relaxation 
and eventual dissipation. ${ }^{27}$ The continuous expression given by Eq. (12) is converted to the matrix representation presented as Eq. (14) via the prescription $\int \mathrm{d} y_{p, q} \rightarrow a_{y_{p, q}} \sum_{n, m}$. The relation $\hbar v_{\alpha}=2 a_{x} t_{x} \sin \left(k_{\alpha} a_{x}\right)$ is used to evaluate the velocities and is obtained from the relation $\hbar v_{\alpha}=\partial E / \partial k_{\alpha}$ using the dispersion relation for a one-dimensional discrete wire, $E=2 t_{x}\left[1-\cos \left(k_{\alpha} a_{x}\right)\right]$.

As an aside, we note that the Green's functions provide a straightforward means of visualizing the electronic behavior within the device as well as enabling the transmission function to be calculated. The local density of states at a grid point $n$, denoted by $[\rho]_{n}$, is related to the imaginary component of $G^{R}$ by the expression ${ }^{27,28}$

$$
[\rho]_{n}=\frac{i}{2 \pi}\left(\left[G^{R}\right]_{n n}-\left[G^{A}\right]_{n n}\right) .
$$

This allows the spatial distribution of the electronic states at any energy to be "visualized," which in certain situations can be a useful aid in understanding the characteristics of the device.

The calculation of the transmission function is all one needs to evaluate the transport quantities of interest, i.e., the electrical conductance $G$ and the thermopower $S$, assuming one is in the linear response regime where the potential and temperature differences between the two reservoirs are sufficiently small. To be specific, $G$ and $S$ are evaluated between the two terminals and in the equations that follow $t$ refers to the transmission function between the two leads, i.e., $t(E)=t_{12}(E)=t_{21}(E)$. The transport coefficients are obtained from $t(E)$ and the derivative of the Fermi function $f_{0}$ evaluated at the global chemical potential $\mu$ and temperature $T$ via the expressions, ${ }^{29,30}$

$$
\begin{gathered}
G=\frac{2 e^{2}}{h} \int_{\epsilon_{1}}^{\infty}\left(-\frac{\partial f_{0}}{\partial E}\right) t(E) \mathrm{d} E, \\
S=-\frac{L}{G}
\end{gathered}
$$

where $L$ is given by

$$
L=-\frac{2|e|}{h T} \int_{\epsilon_{1}}^{\infty}\left(-\frac{\partial f_{0}}{\partial E}\right)(E-\mu) t(E) \mathrm{d} E,
$$

and $\epsilon_{1}$ is given by Eq. (10). These formulae are expected to be valid given that the relaxation mechanisms only operate well away from the primary regime of interest. ${ }^{29}$ Convenient units are $G_{0}=e^{2} / h$ for the conductance, which is the quantum of electrical conductance per spin, and $k_{B} / e$ for the thermopower. As discussed in the Introduction, it is assumed that the electronic component dominates at low temperature and therefore the effects of phonons can be ignored. With this in mind, the thermoelectric figure of merit $Z T$ can be estimated (as a measure of the efficiency of the device in thermoelectric applications ${ }^{31}$ ) using the formula ${ }^{32}$

$$
Z T=\frac{G S^{2} T}{\kappa}=\frac{L^{2} T}{G \kappa} .
$$

Here, $\kappa$ is the thermal conductance defined as

$$
\kappa=-K\left(1+\frac{S^{2} G T}{K}\right)=-K-\frac{L^{2} T}{G},
$$

where

$$
K=-\frac{2}{h T} \int_{\epsilon_{1}}^{\infty}\left(-\frac{\partial f_{0}}{\partial E}\right)(E-\mu)^{2} t(E) \mathrm{d} E
$$

When the transmission function is a slowly varying function of energy, the above results are not particularly interesting, i.e., the thermal conductance $\kappa$ is essentially the same as the electrical conductance $G$ multiplied by a scaling factor $G_{\mathrm{th}} /$ $G_{0}$, with $G_{\text {th }}=k_{B}^{2} \pi^{2} T / 3 h$ being the quantum of thermal conductance per spin. This is a manifestation of the Wiedemann-Franz law. ${ }^{29}$ However, when the transmission function is a rapidly varying function of energy, as it is near resonances associated with a given device geometry (see Sec. III), much richer behavior can be observed. In such cases, enhancement of the thermopower (which can even change sign as a function of chemical potential), together with a breakdown of the Wiedemann-Franz relationship between the electrical and thermal conductance, can lead to a significant increase in the thermoelectric figure of merit. This is one of the principal potential attractions of this type of device structure. ${ }^{17}$

\section{RESULTS AND DISCUSSION}

The transmission functions (or coefficients) for single and double-bend waveguides are presented in Fig. 2. For all calculations, an effective mass $m^{*}=0.067 m_{0}$ representative of GaAs was used and the value of the lead length $d$ was $16 \mathrm{~nm}$. This value was found by comparing results for a number of values of $d$. Beyond $16 \mathrm{~nm}, t(E)$ is insensitive to $d$. However, large values of $d$ also require a large number of grid points, increasing calculation time. The value of $d=16 \mathrm{~nm}$ represents a good tradeoff between accuracy and computational speed. The results are in good qualitative and quantitative agreement with those obtained by other authors. ${ }^{6,7,10}$

The transmission function of the single bend is generally smooth with a cutout region around $22 \mathrm{meV}$ where no electrons can pass through the structure. The addition of a second bend increases the complexity of the transmission function, giving rise to additional peaks and troughs overlaid on the

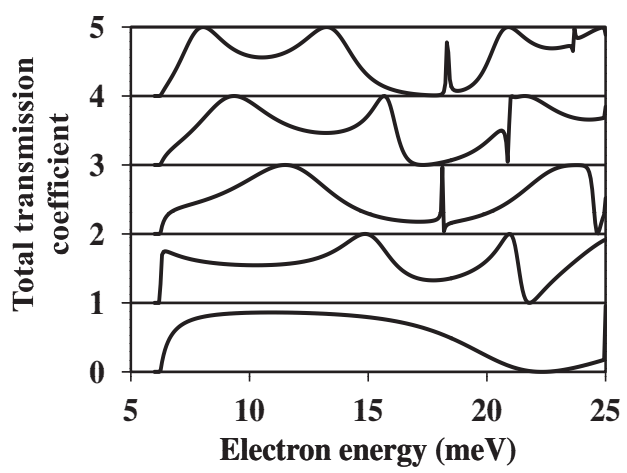

FIG. 2. Transmission plots for five structures, each with $W=30 \mathrm{~nm}$. From bottom to top: a single bend, followed by double bends with $a=10,20,30$, and $40 \mathrm{~nm}$. Curves for different structures are offset for clarity. 
single bend results. This is due to interference between the bends. The shifting positions of the transmission peaks with the interbend length $a$ show that the geometry of the structure can be used to tune the behavior of the device. The maxima in the transmission function occur when the electron wavenumber $k_{\alpha}$ satisfies a standing wave condition in a twodimensional box approximately the length of the section between the bends. ${ }^{4}$

The linear response coefficients for a double bend are shown in Fig. 3. The conductance follows the form of the transmission function closely although the fine detail is lost due to the non-zero temperature. In the regions where the transmission function varies rapidly with energy, the thermopower displays pairs of peaks, one positive and one negative. This is in agreement with the theory that the thermopower is related to the energy derivative of the transmission function. ${ }^{33,34}$ Therefore, the sharper the resonances in the transmission function, the larger the magnitude of the thermopower peaks.

The effect of Anderson disorder ${ }^{35}$ on the behavior of the double bend is now considered. This is a model of a disordered material where a potential, chosen randomly from a uniform distribution in the range $-V_{D}$ to $+V_{D}$ inclusive, is added to each grid point in the device. We choose units of $t_{0}=\hbar^{2} / 2 m^{*} a_{0}^{2}$ for $V_{D}$, with $a_{0}=a_{x}=a_{y}$. The disorder is generated randomly and so variation between samples is expected. Fig. 4 therefore shows the conductance and thermopower for four different disorder configurations at two different values of $V_{D}$.

The conductance is in general reduced by the presence of disorder due to the increased difficulty for an electron to pass through the structure. The peaks in the conductance are also shifted by the disorder in a configuration-dependent way. These changes are reflected in the thermopower, where
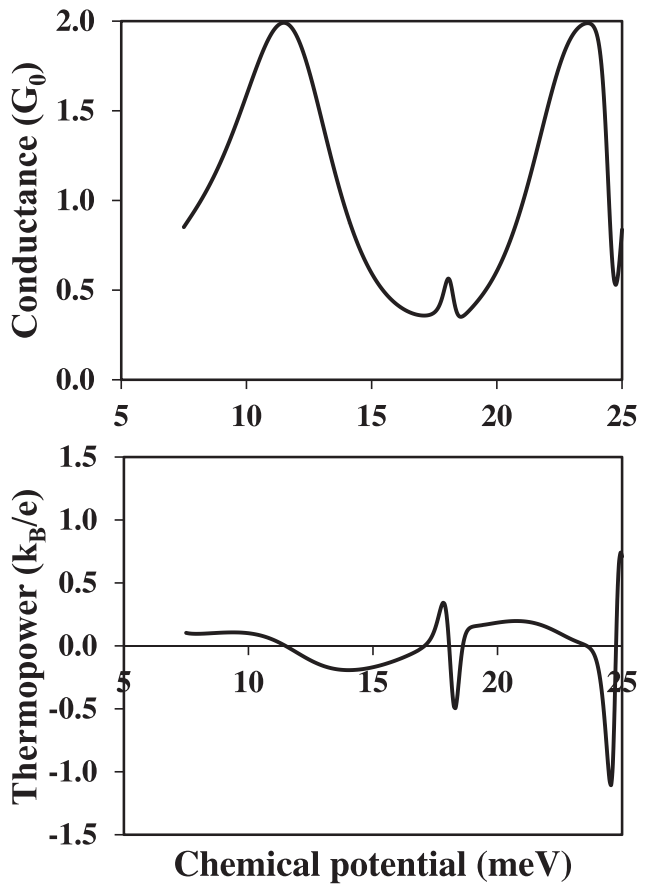

FIG. 3. Linear response conductance $G$ (top) and thermopower $S$ (bottom) for a double bend with parameters $W=30 \mathrm{~nm}, a=20 \mathrm{~nm}$, and thermal energy $k_{B} T=0.125 \mathrm{meV}(T \approx 1.4 \mathrm{~K})$.
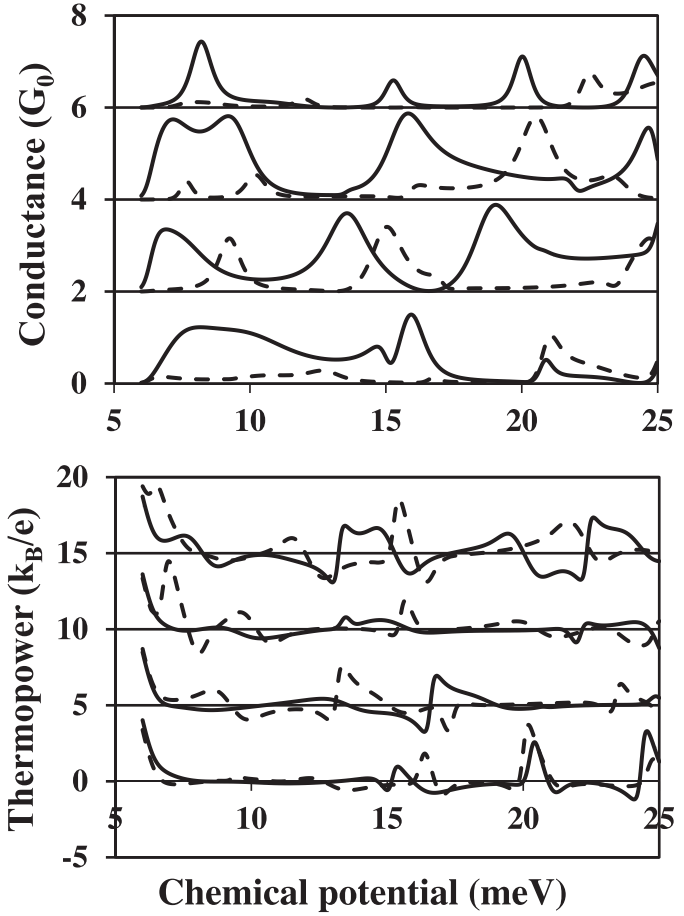

FIG. 4. Linear response conductance $G$ (top) and thermopower $S$ (bottom) for a double bend with parameters $W=30 \mathrm{~nm}, a=20 \mathrm{~nm}$, and thermal energy $k_{B} T=0.125 \mathrm{meV}(T \approx 1.4 \mathrm{~K})$ in the presence of Anderson disorder. The results for four different random disorder configurations are shown, with the curves for different configurations offset for clarity. The disorder strength was $V_{D}=0.3 t_{0}$ (solid curves) and $0.5 t_{0}$ (dotted) with $t_{0}=0.14 \mathrm{eV}$.

the features shift on the energy axis, depending on the configuration and strength of the disorder. Perhaps the most interesting observation is that the presence of disorder can lead to increased values in the thermopower. Coupled with the reduced conductance, this can result in an increase in the thermoelectric figure of merit.

U-bend structures, equivalent to two double bends positioned back to back, are now considered. There are now two interbend lengths $a$ and $b$, which can be used to tune the behavior of the device. Initially, however, we impose the condition that $a=b$ to examine how the additional bends affect the transmission function. This is shown in Fig. 5 where one can see that the addition of the third and fourth bends causes the formation of triplets of peaks in the transmission function. This is in agreement with the prediction

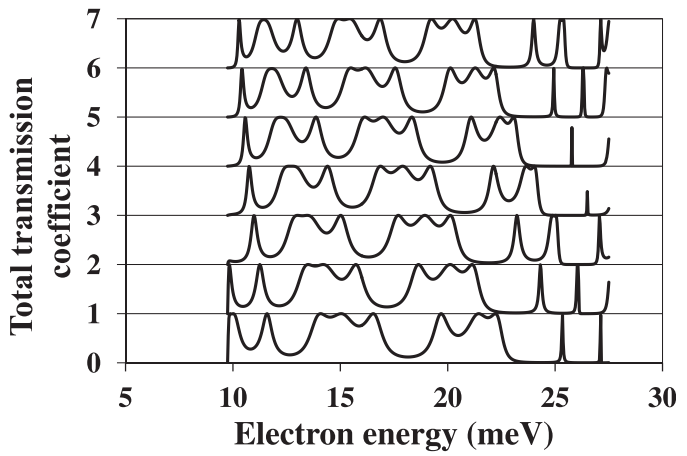

FIG. 5. Transmission plots for U-bend structures with $W=24 \mathrm{~nm}$. From bottom to top, the interbend lengths were $a=b=52,56,60,64,68,72$, and $76 \mathrm{~nm}$; the curves for different structures are offset for clarity. 
that, if a structure has $N_{B}$ bends, there will be $N_{B}-1$ peaks in the transmission function. ${ }^{18}$ The transmission peaks show a very clear progression towards lower energies as the lengths of the sections between the bends are increased, as seen for the double bend. In addition to the relatively broad triplet peaks, fine resonances are apparent in Fig. 5. As discussed above, these can be expected to lead to large magnitudes in the thermopower, as confirmed by Fig. 6. In conjunction with the large values of $S$, the low conductivity in the same energy range means that the thermoelectric figure of merit is large, reaching a maximum of about 11.

The effects of disorder and imperfections in the U-bend structure are now examined. The discretized approach is ideally suited to a model of edge roughness based on that of Todorov and Briggs. ${ }^{20}$ The roughness is specified by the correlation length $L_{\mathrm{cor}}$, variance length $L_{\mathrm{var}}$, and maximum narrowing width $W_{\max }$. Each of these lengths is specified in terms of grid spacings. The edges of the waveguide are split into sections with lengths picked at random from the three values $L_{\mathrm{cor}}-L_{\mathrm{var}}, L_{\mathrm{cor}}$, and $L_{\mathrm{cor}}+L_{\mathrm{var}}$. In alternate sections, an arbitrarily large potential is applied to a strip whose width is chosen randomly between 1 and $W_{\max }$ inclusive. A sample roughness configuration is shown in Fig. 7.

In Fig. 8, the transmission function for a U-bend with four different configurations of edge roughness is presented. These results are compared to those for the unaltered smooth U-bend and also to a waveguide, which has been narrowed by a single continuous row of grid points. The various edge roughness configurations have differing effects on the transmission as we would expect, although the results are qualitatively similar for all of the rough devices. Each of these curves bears a much closer resemblance to that of the
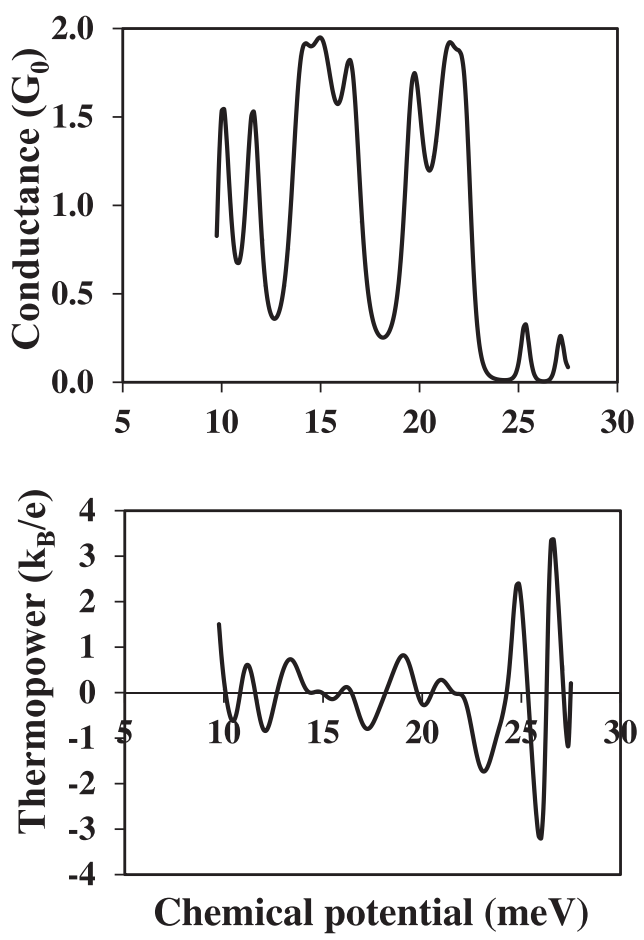

FIG. 6. Linear response conductance $G$ (top) and thermopower $S$ (bottom) for a U-bend with $a=b=52 \mathrm{~nm}$ and $W=24 \mathrm{~nm}$. The thermal energy was $k_{B} T=0.125 \mathrm{meV}(T \approx 1.4 \mathrm{~K})$.

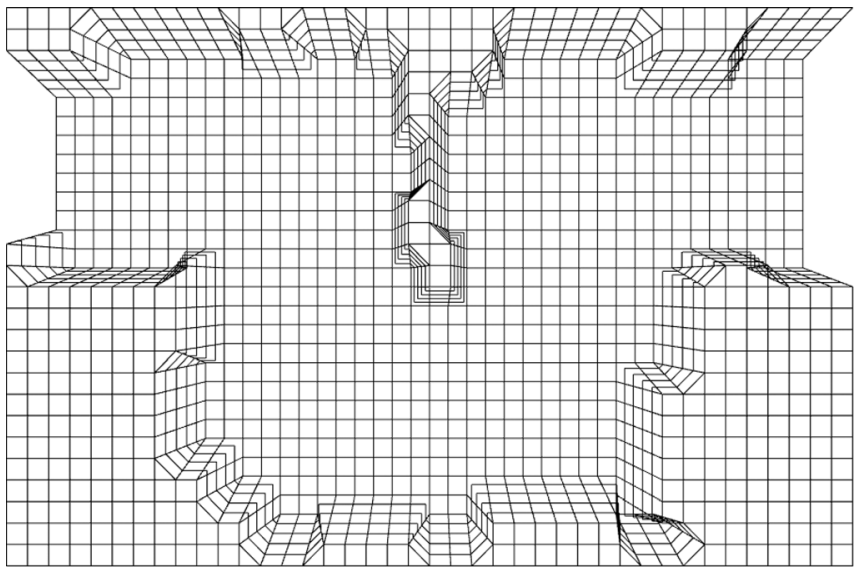

FIG. 7. A sample U-bend with edge roughness applied. The model parameters were $L_{\text {cor }}=5, L_{\mathrm{var}}=2$, and $W_{\max }=2$.

narrowed smooth structure than the unmodified one. This is not surprising since the average narrowing caused by the roughness will be closer to the former than the latter. Overall, the transmission appears to be relatively robust against even a moderately large degree of roughness compared to the results obtained for the narrowed structure. However, the thermoelectric properties will be affected since the details of the transmission peaks can be modified by the presence of edge roughness.

Finally, the impact of Anderson disorder on the performance of the U-bend is examined. Fig. 9 shows the effect of this disorder on the transmission function for a number of random configurations. The transmission peaks can be broadened, shifted, and diminished by the disorder, with the exact effect being determined by the individual configuration and thus difficult to predict. By altering the disorder strength $V_{D}$, the impact of the level of disorder on the behavior of the U-bend is examined. This is shown in Fig. 10. The effect of increasing disorder strength is configuration-dependent with the first configuration in Fig. 10 showing a drop in transmission with increasing $V_{D}$ but little change in the resonant energies. The second configuration shows the reverse of this.

Fig. 11 shows conductance and thermopower results for two of the disorder configurations used in Fig. 9. Due to the

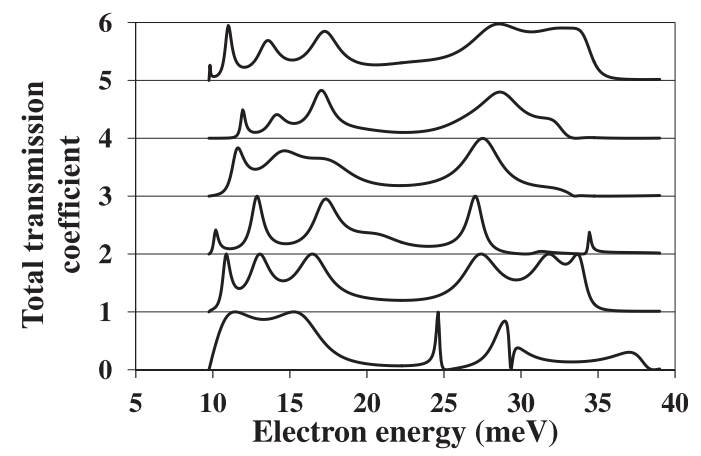

FIG. 8. Transmission plots for U-bends with various edge alterations, with the curves offset for clarity. The parameters were $a=b=2 \mathrm{~nm}$ and $W=24 \mathrm{~nm}$. From bottom to top, the curves are for the original unaltered Ubend, then the narrowed smooth structure, followed by four different random configurations of edge roughness with parameters $L_{\mathrm{cor}}=5, L_{\mathrm{var}}=2$, and $W_{\max }=2$. 


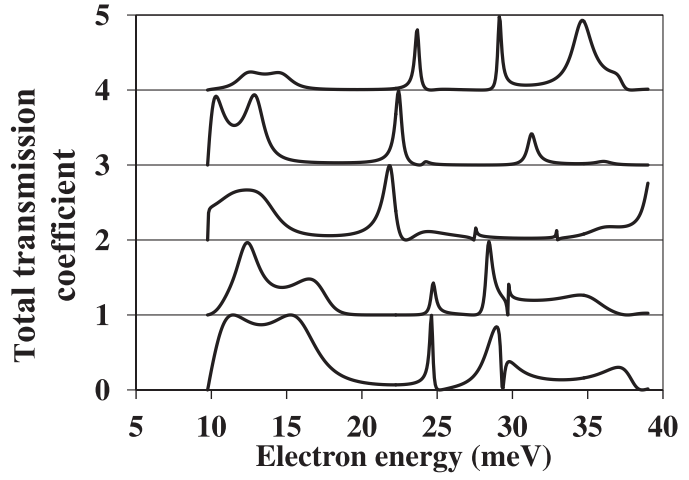

FIG. 9. Transmission plots of a U-bend with $a=b=2 \mathrm{~nm}$ and $W=24 \mathrm{~nm}$ in the presence of Anderson disorder. The lowest curve is for the device in the absence of disorder, whilst the others are for four different random configurations of disorder all at strength $V_{D}=0.3 t_{0}$, with $a_{x}=a_{y}=2 \mathrm{~nm}$ such that $t_{0}=0.14 \mathrm{eV}$.

configuration-dependent nature of the disorder, the details of the two sets of coefficients differ quite significantly although the overall form of the curves is qualitatively similar. The conductance follows the transmission curves closely as expected and consequently reflects the effect of the disorder. Similarly, the thermopower is altered by the change in energy and sharpness of the transmission resonances. As a result, the magnitudes of the thermopower peaks are reduced substantially. These conditions lead to a reduction in the thermoelectric figure of merit for the U-bend, although peaks approaching 2.5 are still seen for the structures presented here.
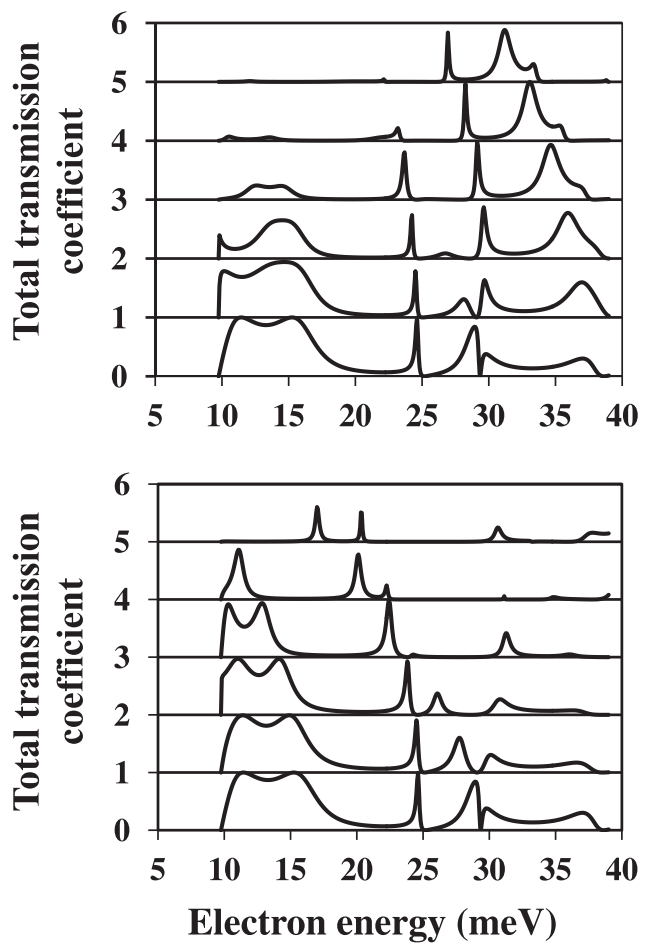

FIG. 10. The effect of different disorder strengths on a U-bend with $a=b=2 \mathrm{~nm}$ and $W=24 \mathrm{~nm}$. From bottom to top, the different curves are for disorder strengths $V_{D}=0,0.1,0.2,0.3,0.4$, and $0.5 t_{0}$, with $t_{0}=0.14 \mathrm{eV}$. The disorder configurations used were as for the top (upper plot) and second from top (lower plot) curves of Fig. 9.
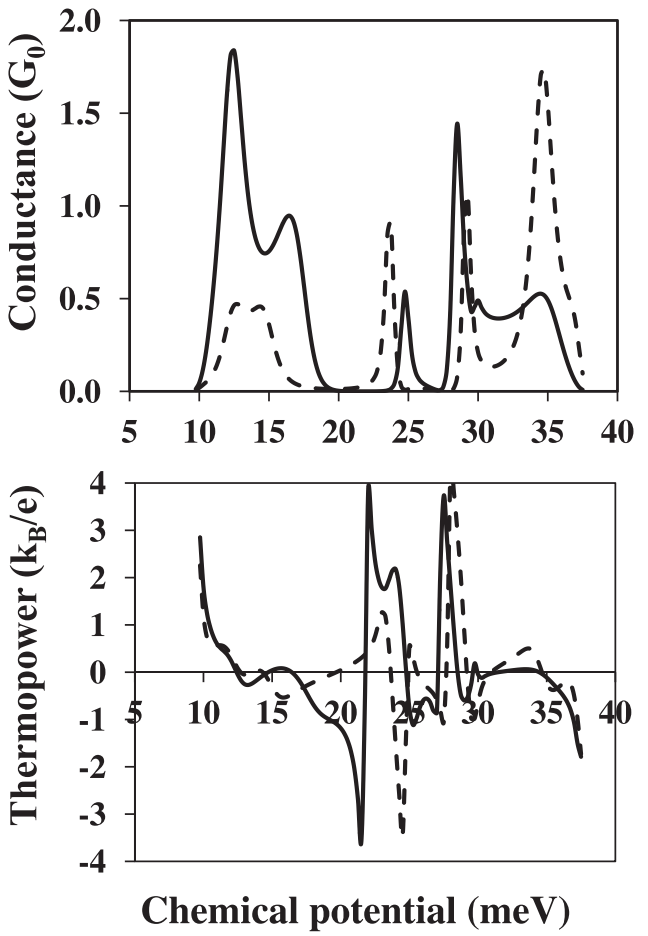

FIG. 11. Linear response conductance $G$ (top) and thermopower $S$ (bottom) for disordered U-bends with $a=b=2 \mathrm{~nm}$ and $W=24 \mathrm{~nm}$. The disorder strength was $V_{D}=0.3 t_{0} \quad\left(t_{0}=0.14 \mathrm{eV}\right)$ and the thermal energy was $k_{B} T=0.125 \mathrm{meV}(T \approx 1.4 \mathrm{~K})$. Results are shown for the bottom (solid curves) and topmost (dotted) disorder configurations used for Fig. 9.

\section{CONCLUSIONS}

In this paper, a quantum transport theory based on Green's functions on a discrete real space grid is presented which allows the calculation of the transmission function, linear response thermopower, and electrical conductance. The theory is applied to electron waveguide structures containing single and double right-angle bends, obtaining good qualitative agreement with results elsewhere in the literature. Stop-band behavior is observed in the transmission function for both of these structures and sharp resonances in the transmission function of double bends are shown to give rise to positive-negative pairs of peaks in the thermopower. The length of the section of waveguide between the two bends determines the energies at which the maxima in the transmission function occur, thus allowing these to be tuned by adjusting the interbend length $a$.

A novel combination of two double bends placed back to back to form a U-bend is studied in depth. The four bends give rise to triplets of peaks in the transmission function, whilst the three interbend sections allow the energies of the transmission peaks to be controlled extensively. Like the double bend, the transmission function of the U-bend also exhibits stop-band behavior and fine resonances in this region give rise to large magnitude peaks in the thermopower. Coupled with the low conductance predicted for these energies, this leads to values of the thermoelectric figure of merit well in excess of unity.

One of the strengths of the numerical approach presented is the ease with which imperfections can be modeled. The introduction of Anderson disorder to the double-bend 
waveguides shows that the thermoelectric figure of merit can increase in disordered systems. Both Anderson disorder and edge roughness models are applied to the U-bend. In these cases, the general structure of the transmission function is robust against a moderate degree of both types of imperfection whilst, if the level of disorder is large, the height, breadth, and energy of the peaks in the transmission function vary in a configuration- and energy-dependent fashion. The linear response coefficients also reflect these changes, with one of the consequences being that the thermopower drops in comparison to a "perfect" structure. As a result, the thermoelectric figure of merit is reduced, though still peaks at values in excess of 2.

From a practical point of view, the U-bend has a potentially compact form and also gives the capability of tuning the chemical potential at which optimal thermoelectric conversion is attained. We conclude that, even when imperfections are taken into account, the U-bend has a strong thermoelectric signature.

\section{ACKNOWLEDGMENTS}

A.J.P. acknowledges the support of Engineering and Physical Sciences Research Council (UK) for financial support through a Doctoral Training Grant.

${ }^{1}$ B. J. van Wees, H. van Houten, C. W. J. Beenakker, J. G. Williamson, L. P. Kouwenhoven, D. van der Marel, and C. T. Foxon, Phys. Rev. Lett. 60, 848 (1988)

${ }^{2}$ D. A. Wharam, T. J. Thornton, R. Newbury, M. Pepper, H. Ahmed, J. E. F. Frost, D. G. Hasko, D. C. Peacock, D. A. Ritchie, and G. A. C. Jones, J. Phys. C 21, 209 (1988).

${ }^{3}$ F. Sols, M. Macucci, U. Ravaioli, and K. Hess, Appl. Phys. Lett. 54, 350 (1989).

${ }^{4}$ A. Weisshaar, J. Lary, S. M. Goodnick, and V. K. Tripathi, Appl. Phys. Lett. 55, 2114 (1989).

${ }^{5}$ J. Wu, M. N. Wybourne, W. Yindeepol, A. Weisshaar, and S. M. Goodnick, Appl. Phys. Lett. 59, 102 (1991).

${ }^{6}$ T. Kawamura and J. P. Leburton, J. Appl. Phys. 73, 3577 (1993).
${ }^{7}$ T. Kawamura and J. P. Leburton, Phys. Rev. B 48, 8857 (1993).

${ }^{8}$ R. Kim, S. Datta, and M. S. Lundstrom, J. Appl. Phys. 105, 034506 (2009).

${ }^{9}$ M. Hannan, R. Grundbacher, I. Adesida, and R. W. Giannetta, J. Vac. Sci. Technol., B 14, 4062 (1996).

${ }^{10}$ J. Zhou and R. Yang, Appl. Phys. Lett. 98, 173107 (2011).

${ }^{11}$ W.-Q. Huang, K.-Q. Chen, Z. Shuai, L. Wang, W. Hu, and B. S. Zou, J. Appl. Phys. 98, 093524 (2005).

${ }^{12}$ F. Xie, K.-Q. Chen, Y. G. Wang, Q. Wan, B. S. Zou, and Y. Zhang, J. Appl. Phys. 104, 054312 (2008).

${ }^{13}$ Y. Qi, Z. Wang, M. Zhang, F. Yang, and X. Wang, J. Mater. Chem. A 1, 6110 (2013).

${ }^{14}$ G. Xie, Y. Guo, X. Wei, K. Zhang, L. Sun, J. Zhong, G. Zhang, and Y.-W. Zhang, Appl. Phys. Lett. 104, 233901 (2014).

${ }^{15}$ K.-M. Li, Z.-X. Xie, K.-L. Su, W.-H. Luo, and Y. Zhang, Phys. Lett. A 378, 1383 (2014).

${ }^{16}$ X. Zianni, P. N. Butcher, and M. J. Kearney, Phys. Rev. B 49, 7520 (1994).

${ }^{17}$ B. Wang, J. Zhou, R. Yang, and B. Li, New J. Phys. 16, 065018 (2014).

${ }^{18}$ H. Xu, Phys. Rev. B 47, 9537 (1993).

${ }^{19}$ G. Kirczenow, Phys. Rev. B 39, 10452 (1989).

${ }^{20}$ T. N. Todorov and G. A. D. Briggs, J. Phys.: Condens. Matter 6, 2559 (1994).

${ }^{21}$ R. Landauer, IBM J. Res. Dev. 1, 223 (1957).

${ }^{22}$ M. Büttiker, Phys. Rev. Lett. 57, 1761 (1986).

${ }^{23}$ M. Büttiker, Y. Imry, R. Landauer, and S. Pinhas, Phys. Rev. B 31, 6207 (1985).

${ }^{24}$ M. Büttiker, Phys. Rev. B 42, 3197 (1990).

${ }^{25}$ S. Datta, Electronic Transport in Mesoscopic Systems (Cambridge University Press, 1997).

${ }^{26}$ D. S. Fisher and P. A. Lee, Phys. Rev. B 23, 6851 (1981).

${ }^{27}$ M. Di Ventra, Electrical Transport in Nanoscale Systems (Cambridge University Press, 2008).

${ }^{28}$ S. Datta, Quantum Transport: Atom to Transistor (Cambridge University Press, 2005).

${ }^{29}$ P. N. Butcher, J. Phys.: Condens. Matter 2, 4869 (1990).

${ }^{30}$ H. van Houten, L. W. Molenkamp, C. W. J. Beenakker, and C. T. Foxon, Semicond. Sci. Technol. 7, B215 (1992).

${ }^{31}$ O. A. Tretiakov, A. Abanov, S. Murakami, and J. Sinova, Appl. Phys. Lett. 97, 073108 (2010).

${ }^{32}$ H. Goldsmid, Thermoelectric Refrigeration, The International Cryogenics Monograph Series (Plenum Press, New York, 1964).

${ }^{33}$ M. Cutler and N. F. Mott, Phys. Rev. 181, 1336 (1969).

${ }^{34}$ P. Streda, J. Phys.: Condens. Matter 1, 1025 (1989).

${ }^{35}$ P. W. Anderson, Phys. Rev. 109, 1492 (1958). 